\title{
Alternative alla detenzione tra net widening e need- risk assessment
}

\author{
di Giuseppe Caputo*
}

L'autore mette in discussione l'idea che l'emergere nel caso italiano di un approccio attuariale al governo dell'esecuzione penale sia conseguenza della crisi del correzionalismo welfarista. Riconduce le pratiche sociogiuridiche di distribuzione dell'utenza tra le diverse opzioni esecutive, apparentemente basate su valutazioni predittive del rischio di recidiva, alle necessità imposte dalla crescente bulimia punitiva e ad una evoluzione della logica welfarista della standardizzazione e dell'allocazione ottimale delle risorse.

Parole chiave: carcere, misure alternative, recidiva, rischio, welfare

Non-custodial measures between net widening and need-risk assessment

The author questions the idea that the emergence in the Italian case of an actuarial approach to the government of criminal justice is a consequence of the crisis of welfarist correctionalism. The socio-legal and administrative practices of distribution of convicted among the various punitive programs, apparently based on recidivism risk assessment, must be considered as a necessity due to the punitive bulimia and an evolution of the welfarist tecniques of standardization and optimal resources' allocation.

Keywords: prison, non-custodial measures, recidivism, risk, welfare

\section{Introduzione.}

A partire dagli anni 60-70 del '900 un articolato movimento critico nei confronti del sistema correzionalista ha contribuito a formare un'immagine negativa del carcere che viene considerato un ambiente patogeno che inevitabilmente induce alla ricaduta nel crimine. Si pensi in particolare agli studi sociologici sulla "società dei detenuti" (Clemmer1941; Sykes 1958; Goff-

*Università degli Studi di Firenze. giuseppe.caputo@unifi.it 


\section{Caputo}

man 1961), alla storiografia revisionista (Rothman 1971; Foucault 1975; Ignatieff 1978) e alla criminologia critica (Lemert 1967; Matza 1969; Baratta 1982) che hanno evidenziato l'inefficacia dell'istituzione carceraria e messo in crisi alcuni dei suoi paradigmi fondativi come quello del trattamento individualizzato delle cause della devianza. Oppure alla critica neoliberale che ha accusato il correzionalismo di essere incapace di abbattere $\mathrm{i}$ tassi di recidiva e ha proposto l'abbandono delle teorie social-preventive in favore di un ritorno a teorie meramente retributive della pena (Von Hirsch 1976).

In questo contesto ha preso le mosse il progetto politico di umanizzazione del sistema punitivo volto in particolare ad evitare le detenzioni brevi per reati minori e a sostituirle con misure non detentive. Il movimento riformista che ne è derivato ha portato ad una articolazione delle modalità esecutive che, pur lasciando al centro dell'orizzonte punitivo la detenzione in carcere, comprendono ora una varietà di misure "alternative" che consentono di scontare tutta o parte della pena fuori dalle mura del carcere. Solo per menzionare le principali misure si pensi all'affidamento in prova (art. 47 o.p.), alla semi-libertà (art. 48 o.p.) e alle varie ipotesi di detenzione domiciliare (artt. 47 ter, quater, quinquies o.p) ${ }^{1}$. A questa tendenza riformista si è di recente sovrapposta quella a prevedere l'uso dei lavori di pubblica utilità al posto della detenzione e/o di pene pecuniarie, secondo il modello del community service anglosassone (Albrecht-Schädler 1986) che si rifà, invece, alle teorie della restorative justice diffuse nel mondo anglosassone sin dalla fine degli anni '70 (Barnett 1977 e Eglash 1977). I "lavori di pubblica utilità" (lpu), oltre che per i reati di competenza del giudice di pace, sono applicabili come misura sostitutiva della detenzione ai tossicodipendenti che commettono reati di droga di "lieve entità", a coloro che guidano in stato di ebrezza o sotto l'effetto di stupefacenti (artt. 186-187 cds) ${ }^{3}$. Sono previsti poi all'interno del nuovo istituto della messa alla prova $(\operatorname{map})^{4}$ che prevede la sospensione del processo, con conseguente estinzione del reato, subordinata all'esecuzione da parte dell'imputato di un programma di attività ${ }^{5}$.

\footnotetext{
${ }^{1}$ L. 26 luglio 1975, n. 354 sull'Ordinamento penitenziario (o.p.)

${ }^{2}$ Art. 73.5 bis, 1.9 ottobre 1990, n. 309 introdotto dal d.1. 20 marzo 2014, n. 36

${ }^{3}$ L. 29 luglio 2010, n. 120.

${ }^{4}$ Art. 168 bis, ter e quater c.p. introdotti con la 1.28 aprile 2014, n. 67.

${ }^{5}$ In buona parte esse sono mutuate da quelle dell'affidamento (art. 47 o.p.)
} 


\section{Caputo}

Si commetterebbe, però, un grossolano errore nel considerare tale movimento, avviato in Italia con l'Ordinamento penitenziario del 1975 e la legge "Gozzini" del $1986^{6}$, come una semplice fuga dal carcere. Le misure alternative alla detenzione hanno rappresentato, infatti, anche un nuovo atto di fede verso la capacità del sistema punitivo correzionalista di far fronte al mandato di controllo della devianza e della criminalità e di riuscire a mantenere, con nuovi strumenti, quella promessa di ridurre la recidiva sempre disattesa dal carcere. Il contesto in cui esso viene introdotto, non bisogna dimenticarlo, è quello dell'affermazione di una cultura welfarista di gestione della devianza e del conflitto sociale che ha determinato uno spostamento d'interesse dal reato alle condizioni sociali e psicologiche dell'autore (Garland 1985). Il paradigma riabilitativo dell'o.p. del 1975 si basa, infatti, su dispositivi di diagnosi delle carenze soggettive dei condannati - di natura sociale o fisio-psichiche - che possono essere ricondotte a "bisogni" standardizzati di trattamento clinico oppure sociale, da effettuarsi in carcere o all'esterno.

In sintesi, l'attuale articolato sistema delle alternative ha assorbito nel corso degli anni una pluralità di funzioni distinte ma complementari. Quella di ridimensionare il ruolo dell'istituzione carceraria che sarebbe dovuta diventare luogo residuale per il trattamento terapeutico degli individui portatori di una maggiore pericolosità sociale, sulla base di una diagnosi basata sulla "osservazione scientifica della personalità". Quella di favorire percorsi di reinserimento sociale mediante l'implementazione di programmi ideati a partire dallo studio dei bisogni individuali. Quella di sostituire il carcere con programmi aventi finalità riparativa e/o di mediazione penale. Sullo sfondo la grande promessa della penalità moderna di contenere la ricaduta nel crimine.

Nelle pagine che seguono intendo mostrare come l'apparato dell'esecuzione penale si sia adattato a queste mutevoli esigenze ed orientamenti criminologici. Un primo adattamento, che definiremo primario, è dipeso dalla ripresa del processo di carcerizzazione che ha indotto ad usare le alternative alla detenzione per evitare che la nuova bulimia punitiva ricadesse sul solo sistema carcerario, favorendo un fenomeno di net widening (Cohen 1979). Il secondo adattamento, che definiremo di tipo secondario, è conseguenza dell'evoluzione della cultura welfarista inclusivista in favore di una nuovo modello di gestione del rischio di recidiva, che ha investito

\footnotetext{
${ }^{6}$ L. 10 ottobre 1986, n. 663.
} 


\section{Caputo}

anche le pratiche punitive e condizionato la cultura degli operatori sociali e giuridici. Il mandato oggi percepito dalla burocrazia dell'esecuzione penale non è più solo quello di pianificare interventi terapeutici o assistenziali volti a soddisfare i bisogni dei devianti, ma anche quello di selezionarli e differenziarli per gruppi di "rischio" e distribuirli tra numerose opzioni esecutive.

Vedremo che tale tendenza è normalmente ricondotta alla crisi del paradigma riabilitativo e al riemergere dell'approccio liberale retributivo al fenomeno della devianza, in conseguenza della crisi del welfare state e della cultura correzionalista (Garland 2001). Sosterremo che tale interpretazione, sviluppata nel contesto anglosassone, mal si adatti al caso italiano. In primo luogo, perché i criteri di need-risk assessment cui si informano i nostri attori dell'esecuzione penale sembrano mutuati dalla logica attuariale dei programmi di welfare e, dunque, possono essere considerati come una naturale evoluzione dell'approccio correzionalista. In secondo luogo, tale interpretazione non spiega le più recenti evoluzioni delle pratiche giuridico-sociali dell'esecuzione penale italiana, che non sembra affatto essere stata investita da quella cultura managerialista che altrove ha portato ad elaborare strumenti analitici di predizione della recidiva (Feeley-Simon 1992). Nel caso italiano si sono creati, piuttosto, dispositivi di distribuzione dell'utenza del sistema penale non riconducibili a dispositivi di giustizia attuariale ma a criteri di profiling prodotti per via giurisprudenziale e legale oppure per via esperienziale dagli operatori dell'esecuzione penale.

\section{Bulimia punitiva e misure alternative}

L'introduzione nel nostro ordinamento delle misure alternative con la 1. $354 / 75$ è coincisa con un processo ri-carcerizzazione, in controtendenza con il trentennio precedente durante il quale si era verificato un processo di forte decarcerizzazione (Pavarini 1997). L'analisi dei dati relativi alla detenzione e alle misure alternative mostra che l'aumento progressivo dell'uso delle alternative al carcere abbia assecondato e non attenuato questo trend di incarcerazione. 


\section{Caputo}

Dal 1976 il tasso di detenzione è raddoppiato passando da 51 detenuti per 100.000 abitanti fino a toccare il punto massimo di 110 nel $2010^{7}$. In questo intervallo di tempo le cicliche diminuzioni del tasso sono direttamente imputabili a numerosi provvedimenti clemenziali. Anche se il più recente ciclo deflattivo, verificatosi nel biennio 2014-2015, è frutto di una serie di provvedimenti temporanei - come la liberazione anticipata specia$1 e^{8}$ - adottati dal Governo italiano a seguito della condanna della Corte Edu nel caso Torreggiani et al. c. Italia per il sovraffollamento del sistema penitenziario (Caputo-Ciuffoletti 2017)

Il processo di carcerizzazione è prodotto alternativamente o congiuntamente - a seconda degli anni- da due diversi fenomeni. Il primo è la tendenza ad un maggior ricorso alla repressione penale. Il secondo è quello dell'aumento della severità delle condanne penali. Dal 1976 al 1990 si è verificato un aumento del tasso di detenzione prodotto dal saldo negativo tra usciti ed entrati nonché dal numero delle condanne che aumentano del $+52 \%{ }^{9}$. Dopo la parentesi 1984-1990, in cui il tasso è stato contenuto anche grazie ad una serie di provvedimenti clemenziali, i primi anni '90 sono stati caratterizzati da un'ulteriore crescita del saldo ingressi/uscite e delle condanne che aumentano del $+161 \%$ tra il 1990 e il 2000. Negli anni successivi, a fronte di una stabilizzazione della variabile condanne e degli ingressi, il tasso di detenzione ha continuato a crescere a causa dell'aumento della lunghezza media della permanenza in carcere che è passata da 6,8 mesi del 1998 ai 13,7 mesi del $2014^{10}$.

I dati sulle misure alternative possono confermarci l'ipotesi sul net widening che abbiamo anticipato. Per ovviare alle diverse modalità con cui $\mathrm{i}$ dati relativi alla detenzione e quelli relativi alle alternative sono raccolti ${ }^{11}$, abbiamo elaborato la variabile dei "detenuti nel corso dell'anno" e l'abbiamo raffrontata con quella analoga dei condannati in misura alterna-

\footnotetext{
${ }^{7}$ Elaborazione dati Istat. Serie storiche. www.istat.it

${ }^{8}$ Con il d.l 146/2013 si erano temporaneamente portati i giorni di liberazione anticipata da 45 a 75 per ogni semestre di buona condotta.

${ }^{9}$ Elaborazione dati Istat. Serie storiche. www.istat.it.

${ }^{10}$ Elaborazione dati Coe. Space I. Anni 1999-2015. www.wp.unil.ch/space/

${ }^{11}$ Per la prima si raccoglie la variabile di stock dei presenti alla data del 31 dicembre o quella di flusso degli ingressi nell'anno. Per le seconde quelle di flusso delle misure in carico e pervenute nell'anno (Ministero della giustizia. Statistiche. www.giustizia.it)
} 


\section{Caputo}

tiva nell'anno ${ }^{12}$. Dal confronto tra le due variabili emerge che negli anni ' 90 e '00 la popolazione incarcerata nel corso dell'anno si è mantenuta tra $\mathrm{i}$ 120.000 e i 140.000 circa (salvo che per gli anni immediatamente successivi all'indulto del 2006), mentre quella sottoposta a misure alternative conosceva un primo boom, passando dalle poche migliaia degli anni '80 fino ai 50.000 . Solo a partire dal 2013 si è verifico un calo della popolazione detenuta di circa 20.000 unità (che va ad assestarsi sui 100.000 all'anno), ma che non è imputabile all'aumento delle misure alternative, che in quegli anni restano costanti tra le 55.000 e le 58.000 unità circa, bensì alle misure deflattive temporanee come la liberazione anticipata speciale.

Tale effetto di net widening risulta evidente anche se confrontiamo le variabili di stock del numero di persone in misura alternativa e in detenzione alla data del 31 dicembre, per il periodo 1997-2017. Nel periodo considerato si può osservare che l'utenza complessiva del sistema penale (carcerario e non) ha subito un boom impressionante, se nel 1997 era complessivamente di 60.000 unità circa, nel 2017 era di 108.010, grazie in particolare alle nuove misure dei lavori di pubblica utilità e della messa alla prova. Nel periodo compreso tra il 2010 (anno di introduzione degli lpu) e il 2017 le misure alternative sono raddoppiate, infatti, passando da 14.274 a $30.505: \mathrm{i}$ lavori di pubblica utilità da 38 del 2010 a 7.120 del 2017, mentre le messe alla prova da 503 del 2014 a 10.760 del 2017. Nel 2017 il sistema dell'esecuzione penale risultava così articolato: $1 p u$ il $7 \%$, messa alla prova il $10 \%$, misure alternative il $28 \%$ e il carcere il $53 \%$.

Questa tendenza all'espansione del controllo penale ha raggiunto oggi un picco che ha solo due precedenti nella storia italiana ${ }^{13}$. Il primo si era verificato nel 1874, quando si è registrato il numero massimo di detenuti (stock): 74.675 con un tasso di detenzione di 262 detenuti per 100.000 abitanti. Nel 1928 si era avuto un nuovo picco con 64.709 detenuti e un tasso di detenzione di 163. Mentre nel 2017, se sommiamo misure alternative,

\footnotetext{
${ }^{12}$ Variabile ottenuta dalla somma degli ingressi nell'anno e dei presenti al 31 dicembre dell'anno precedente. Mentre quella delle misure nell'anno è elaborata dal Ministero come somma delle misure pervenute nell'ano e di quelle in carico al 1 gennaio. Con riguardo al numero di detenuti entrati nell'anno è importante precisare che dal 1998 vengono conteggiati anche gli eventuali reingressi dello stesso soggetto.

${ }^{13}$ Variabili stock al 31 dicembre.
} 


\section{Caputo}

sanzioni sostitutive, messa alla prova e detenzione, abbiamo un picco senza precedenti: 108.010 persone, con un tasso di 179 per 100.000 abitanti.

Questi dati sembrano confermare l'ipotesi già formulata da Cohen (1979), secondo cui l'articolazione dell'esecuzione penale verso modalità meno afflittive tende a favorire una espansione e non una contrazione della rete del controllo penale. Nel caso italiano l'introduzione di misure di controllo penale "leggere" per un verso ha favorito i processi di criminalizzazione primaria facilitando l'introduzione di nuove fattispecie di reato per condotte dotate di minor disvalore sociale, per altro verso ha incrementato la criminalizzazione secondaria spingendo gli attori del sistema penale ad inasprire le attività repressive. Così ad una minore severità del sistema sanzionatorio è coincisa una sua nuova espansione orizzontale.

Questo fenomeno risulta evidente se si osservano le più recenti riforme che hanno esteso l'uso dei lavori di pubblica utilità e introdotto l'istituto della messa alla prova. I lavori di pubblica utilità, infatti, sono normalmente applicati come sanzione alternativa per nuovi reati che vanno a sanzionare condotte, come quelle legate alla guida in stato di ebrezza o alla detenzione di droga di lieve entità, prima punite per via amministrativa. Nel caso della messa alla prova, invece, ci troviamo apparentemente di fronte ad una nuova modalità esecutiva, applicabile alla generalità dei reati minori, adottato con intenti deflattivi ma che, come visto, ha finito per produrre effetti di segno opposto ${ }^{14}$. In proposito possiamo ipotizzare che, come già avvenuto per le misure alternative, l'introduzione di sanzioni percepite come più "dolci" potrebbe aver indotto le agenzie repressive ad estendere la loro rete di controllo a condotte di minor allarme sociale prima ritenute immeritevoli di essere sanzionate o per le quali si tendeva, in ogni caso, ad applicare l'istituto della sospensione condizionale della pena ${ }^{15}$. La map, poi, da strumento deflattivo "dolce" ha comportato paradossalmente anche una espansione qualitativa del controllo penale: la sua applicazione comporta per un semplice imputato l'adempimento di una serie di prescrizioni limitative

${ }^{14} \mathrm{Si}$ ricordi che è stato introdotto insieme ad un pacchetto di misure deflattive seguite alla condanna della Corte Edu per sovraffollamento.

${ }^{15} \mathrm{Si}$ tenga a mente che la sospensione condizionale si applica esclusivamente a individui non recidivi condannati alla reclusione non superiore a 2 anni (art. 163164). La map, applicandosi per reati per cui il limite edittale sia non superiore nel massimo a 4 anni, finisce per riguardare una casistica in larga parte coincidente con essa. 


\section{Caputo}

della libertà personale ${ }^{16}$, ben più restrittive di quelle previste per l'affidamento in prova che, invece, si applica ai soggetti condannati, certamente ben più costrittive di quelle che deriverebbero dalla sospensione condizionale. Il fatto poi che la map si applichi prima ancora di una condanna penale, induce a farle percepire come più certa ed economica: oltre ad evitare il processo, riduce i costi della fase esecutiva che, non solo non avviene in carcere, ma normalmente consiste in programmi di attività sociale gestiti dal terzo settore a costo zero per lo Stato.

\section{La polifunzionalità delle misure alternative tra bisogno, ri- schio e meritevolezza}

Come anticipato la seconda mutazione del progetto correzionalista delle misure alternative è figlio di un adattamento della cultura welfarista ad un paradigma di controllo della devianza e della criminalità basato sulla possibilità di recidiva e non più solo sui "bisogni". Secondo la lettura dominante in larga parte della sociologia e criminologia critica tali nuove strategie di gestione del rischio sarebbero state indotte dalla crescita della domanda di punitività verso le agenzie di controllo istituzionale (Roberts et al. 2003; Pratt 2007) e andrebbero lette alla luce di una della crisi della governamentalità welfarista (Garland 2001). Castel (1991, p. 294) ha sottolineato che è stata proprio la crisi del welfare a favorire politiche basate su nuove strategie di gestione della devianza che non si limitano più alla mera repressione incapacitante degli elementi "indesiderati" (approccio liberale) o ad interventi correttivi o terapeutici (approccio correzionalista), piuttosto la nuova tendenza è quella ad assegnare "destini sociali diversi ai singoli in linea con la loro diversa capacità di soddisfare le esigenze di competitività e redditività". Superata quella "connessione che tra controllo sociale e patologia" (Santoro 2005, p. 95), la nuova penologia attuariale tende ad ignorare i bisogni e le carenze individuali dei devianti per concentrarsi sui delinquenti in forma aggregata da gestire secondo categorie di rischio (Feeley-Simon 1992). In conseguenza di tali evoluzioni, gli attori del sistema penale tenderebbero oggi a distribuire l'utenza secondo la logica cost/benefit riservando

\footnotetext{
${ }^{16}$ Oltre a tutte le prescrizioni già previste per l'affidamento si aggiungono condotte riparative e i lpu.
} 


\section{Caputo}

il carcere, con finalità meramente neutralizzanti, alle categorie a maggior rischio e limitandosi a forme controllo più blande per le altre (FeeleySimon 1992; Pavarini 2000).

Nel caso italiano, a ben vedere, si rinvengono tracce contraddittorie che consentono di confermare solo in parte questo genere di lettura. L'ideologia penale neo-liberale con la sua retorica penal-populista non sembra essere stata in grado di determinare in maniera costruttiva le pratiche di trattamento dei condannati, limitandosi a produrre una espansione quantitativa dell'apparato repressivo e ad introdurre regimi afflittivi speciali. Per tale ragione si è verificato un fenomeno inaspettato di adattamento delle agenzie di penal welfare che si sono formate una propria cultura, volta a gestire in maniera razionale ed economica la bulimia punitiva che abbiamo analizzato, ma che non sembra affatto essere basata sul ricorso a tecnologie di tipo attuariale. Cerchiamo ora di comprendere la natura e le forme di queste nuove pratiche.

Iniziamo con l'osservare che l'utenza del sistema punitivo è gestita mediante programmi meramente contenitivi oppure programmi con una proiezione verso il sistema delle alternative. La scelta tra le due opzioni trattamentali avviene sulla base di due diversi dispositivi di selezione e distribuzione. Il primo, di natura legale ed amministrativa, è dato dall'insieme di previsioni che prevedono trattamenti differenziati per i condannati "immeritevoli"; il secondo è dato da un complesso di criteri giurisprudenziali e di pratiche elaborate dagli attori penali volti alla differenziazione/esclusione dalle misure alternative e/o dal trattamento rieducativo di alcune tipologie di detenuti ritenuti "inaffidabili" sulla base di una valutazione costi/benefici basata su meri criteri esperienziali ${ }^{17}$.

I dispositivi legali di esclusione hanno fatto la loro comparsa quasi contestualmente alla stessa adozione dell'ordinamento penitenziario. Per tale ragione siamo indotti a ritenere che essi non possono essere considerati come conseguenza della crisi paradigma rieducativo. Sin dalle origini, infatti, il progetto dell'ordinamento penitenziario - formalmente basato sulla logica della standardizzazione dei bisogni - ha sviluppato tecniche di differenziazione del trattamento di alcune categorie di devianti sulla base della

${ }^{17}$ Tali pratiche sono state oggetto di una indagine qualitativa che ha riguardato alcuni dei principali attori dell'esecuzione penale e che ho svolto nell'ambito del Progetto di ricerca Crime repression costs in context https://cordis.europa.eu/project/rcn/84109_en.html. 


\section{Caputo}

mera commissione di alcune tipologie di reato oppure perché ritenute disturbanti per l'ordine interno del carcere. Prima attraverso l'istituzione delle sezioni di massima sicurezza (art. 90 o.p.) e il regime del 41 bis o.p., poi mediante la moltiplicazione per via amministrativa dei circuiti penitenziari, come le sezioni di alta sicurezza ${ }^{18}$ o quelle destinate a detenuti vulnerabi$1 i^{19}$.

Nella gran parte di questi casi l'applicazione di forme di controllo particolarmente contenitive non si basa su una valutazione predittiva della recidiva fondata sull'analisi dei bisogni o delle carenze individuali (approccio clinico) o sulla base dell'appartenenza a gruppi considerati di rischio (approccio attuariale). Queste strategie di differenziazione sembrano tutt'oggi rispondere ad un logica meramente retributiva di gradazione del livello di afflittività della punizione in base alla gravità del fatto, non della pericolosità soggettiva che non è mai valutata ma è presunta. Alla stessa ratio sembrano rispondere molte delle esclusioni dall'accesso alle misure alternative previste dall'art. 4 bis o.p. che colpiscono numerose categorie di reati che suscitano particolare allarme sociale.

Gli ulteriori dispositivi di selezione e distribuzione dell'utenza del sistema penale si basano, invece, su requisiti di affidabilità elaborati dalla giurisprudenza dei tribunali di sorveglianza. Generalmente l'ammissione alle misure alternative è subordinata, oltre che alle condizioni formali previste dall'ordinamento penitenziario, anche dal possesso di talune qualità soggettive ritenute imprescindibili garanzie di affidabilità, quali la disponibilità di un lavoro, di un reddito, di una abitazione e di una rete sociofamiliare. Questa prassi fa si che i condannati a pene brevi provvisti di un adeguato capitale sociale accedano alle misure non carcerarie già dalla libertà, grazie al meccanismo di sospensione della pena previsto dall'art. 656 c.p.p. Mentre quelli considerati inaffidabili dovranno invece eseguire la loro condanna in carcere. Questa tendenza è evidenziata dal fatto che solo nel $34 \%$ dei casi si accede alle misure a partire dallo stato di detenzione, mentre nei restanti casi lo si fa dalla libertà.

Tale dispositivo di selezione e distribuzione si regge poi sulle pratiche degli operatori dell'esecuzione penale che si trovano a governare un popolazione detenuta composta da condannati per reati ostativi, condannati a

${ }^{18}$ DAP, Circolare 21 aprile 2009, 3619/6069 e Circolare 5 maggio 2015, 0157181.

${ }^{19}$ Come quelle per sex offenders e i trans-gender (DAP, Circolare 02 maggio 2001, 500422) 


\section{Caputo}

pene lunghe oppure a pene brevi ma già ritenuti non affidabili dalla magistratura di sorveglianza perché recidivi o privi di un capitale sociale adeguato. Entrano a questo punto in gioco una serie di ulteriori criteri di classificazione informale dei detenuti volti a consentirne una gestione razionale. Lo staff trattamentale effettua, in prima battuta, un'attività di need-risk assessment volta ad individuare detenuti appartenenti a categorie "affidabili" (Torrente 2014) tra quelli dotati di un capitale sociale e di uno status legale tale da consentire un eventuale accesso alle misure alternative. Solo per questi il welfare penitenziario potrà assumere ancora le vesti di agenzia di inclusione sociale coerentemente con il mandato rieducativo che, perlomeno in tali casi, continua ad orientare le pratiche degli operatori. Ma si presti attenzione al fatto che, in realtà, anche nel caso dei detenuti inaffidabili la cosiddetta osservazione personologica non viene abbandonata, piuttosto subisce un mutamento di oggetto e di scopo. L'obiettivo non è una predizione delle future condotte extra-murarie, bensì quella della condotta interna al carcere al fine di consentire l'adozione di tutte le più appropriate misure volte a garantire il contenimento della violenza e a prevenire il rischio di evasioni ${ }^{20}$.

A fare le spese di tali prassi giurisprudenziali e amministrative sono in particolare due categorie di condannati etichettati come "inaffidabili", $i$ tossicodipendenti e gli stranieri, per i quali si presume una possibilità di recidiva non contenibile con le misure alternative. I tossicodipendenti, che costituiscono quasi $1 / 3$ della popolazione detenuta, sono infatti tendenzialmente trattati in carcere piuttosto che in libertà all'interno di comunità di recupero $^{21}$. Per quanto riguarda gli stranieri, invece, l'etichettamento deriva dal fatto che essi sono considerati de iure non reinseribili e, dunque, non meritevoli di un investimento di risorse perché destinati all'espulsione a fine pena. Non è un caso che gli stranieri, pur essendo il 31-33\% dei condannati e il $34 \%$ dei detenuti, rappresentino solo il 19\% del totale dei soggetti in misura alternativa, alla quale raramente accedono dalla libertà: sono appena il $15 \%$ degli affidamenti ordinari e solo il $6 \%$ degli affidamenti terapeutici dalla libertà ${ }^{22}$.

${ }^{20} \mathrm{Si}$ veda in proposito la Circolare Dap 25 novembre 2011, 0445330.

${ }^{21}$ Non a caso nel 2017 il 70\% degli affidamenti terapeutici erano concessi a condannati provenienti dalla detenzione, in proporzione esattamente contraria a quella dell'affidamento ordinario.

${ }^{22}$ Elaborazione dati Ministero della giustizia. Statistiche. www.giustizia.it. 


\section{Caputo}

Nel caso italiano, dunque, il nuovo paradigma penologico del "rischio" contrapposto a quello del "bisogno" - che normalmente viene connesso alla crisi del welfarismo penale sembra in realtà porsi ancora in continuità con esso, avendone mutuato quelle tecniche di governo basate sulla standardizzazione delle categorie sociali e sull'allocamento ottimale delle risorse e degli investimenti. Anche se queste pratiche sovvertono in parte il senso originario delle previsioni del modello correzionalista, secondo cui il trattamento carcerario dovrebbe consistere in interventi proattivi di analisi dei bisogni e di facilitazione del reinserimento sociale e non in una mera attività di selezione dei detenuti meno bisognosi su cui concentrare risorse e investimenti.

Il fatto che l'accesso alle misure sia di fatto riservato ad individui dotati di un buon capitale sociale e selezionati in ragione della minore possibilità di ricaduta nel delitto comporta, naturalmente, che il loro tasso di recidiva sia più basso di quelli che scontano la pena in carcere. Quest'ultima conclusione impone di mettere in discussione l'argomento - frequentemente usato nei discorsi pubblici - secondo cui le misure alternative andrebbero preferite al carcere in virtù della loro capacità di contenere la recidiva. E' il caso ad esempio di una delle poche ricerche condotte sull'argomento che ha evidenziato quanto il tasso di recidiva dei soggetti in affidamento in prova al servizio sociale sia molto più basso di quello dei detenuti: il 19\% dei primi contro il 68,45\% dei secondi (Leonardi 2007). La ricerca non è in grado di chiarire, però, se il minor tasso di recidiva sia dovuto alla maggiore efficacia della misura alternativa rispetto al carcere o, come noi ipotizziamo, dovuto al fatto che il sistema delle misure alternative tende a selezionare individui affidabili. Un limite simile è presente anche in un'altra ricerca condotta sul carcere di Bollate secondo la quale regimi detentivi aperti e l'accesso al lavoro all'esterno del carcere sarebbero in grado di abbassare la recidiva di quasi il 50\% (Mastrobuoni-Terlizzese 2014). Gli stessi autori hanno evidenziato che questo dato è probabilmente sovrastimato a causa del fatto che il trattamento avanzato è riservato a detenuti provenienti da altri istituti e "selezionati" tra quelli che hanno dato maggior prova di buona condotta. Quest'ultima ipotesi è parzialmente confermata da una indagine che, nel misurare l'impatto dell'affidamento sulla recidiva, ha preso in considerazione anche lo status economico-occupazionale dei condannati concludendo che, in effetti, la gran parte degli affidati è in possesso di una occupazione stabile (Santoro-Tucci 2006). Per confermare la nostra ipotesi 


\section{Caputo}

si renderebbe necessario, però, anche una comparazione tra i profili giuridici e socio-economici di individui detenuti e in misura alternativa.

\section{Conclusione}

Le strategie di esclusione selettiva che abbiamo sommariamente analizzato potrebbero apparire come una fuga da quell'approccio clinico basato sulla diagnosi e il trattamento dei bisogni, in favore di un approccio di risk assessment della recidiva. Questa interpretazione va però rigettata. In primo luogo perché manca del tutto un sistema di raccolta e di analisi dei dati relativi alla recidiva che costituirebbe la precondizione per l'esercizio di una funzione del genere. E' stato evidenziato che, in paesi come gli Stati Uniti, la valutazione del rischio di recidiva si è imposta dapprima come mera valutazione professionale dello staff della prigione, per poi essere codificato in elaborati modelli attuariali (James 2018) di cui però non v'è traccia nel sistema italiano ${ }^{23}$.

In secondo luogo va chiarito che queste pratiche sembrano essersi affermate non tanto come reazione alla retorica penal-populista e al neoretributivismo, bensì come un portato, contraddittorio certo, dello stesso paradigma correzionalista. Il modello trattamentale dell'individualizzazione non è in effetti mai stato in grado, sin dal momento della sua implementazione, di orientare l'azione degli operatori penitenziari nel fronteggiare $\mathrm{i}$ bisogni di una popolazione detenuta percepita come problematica e composta prevalentemente da persone marginali (Bandini - Gatti 1980). In secondo luogo, il progetto inclusivista basato sulla standardizzazione dei bisogni sin da subito si è mostrato inadeguato nel consentire il governo di una prigione in perenne emergenza sovraffollamento ${ }^{24}$ e dominata da una cultura securitaria ostile alle riforme. Così si è finito per ricorrere ad un complesso meccanismo di valutazione predittiva della condotta intramuraria e/o della recidiva basata su mere prassi esperienziali dei principali attori dell'esecuzione penale. Il metodo clinico di analisi dei bisogni e delle ca-

23 Solo nell'ultima relazione del Ministero della giustizia per l'Inaugurazione dell'anno giudiziario (2017) si annunciava il finanziamento di un progetto pilota che si muove in questa direzione.

${ }^{24}$ In tal senso si leggano la posizione dei vertici dell'amministrazione penitenziaria all'indomani della riforma Gozzini (Daga 2008: 20). 


\section{Caputo}

renze, lungi dall'essere abbandonato, si è trasformato in criterio di definizione di destini sociali dei condannati sulla base della capacità reddituale $\mathrm{e}$ lavorativa. Le "relazioni di sintesi" prodotte dallo staff dell'esecuzione carceraria sono divenute una certificazione di idoneità per l'accesso alle alternative. Il correzionalismo è così caduto in una profonda contraddizione: l'analisi dello status socio-economico del condannato da strumento di definizione dei bisogni, è divenuto patente di meritevolezza per l'accesso ad una punizione più dolce. Anche se, va detto, non si tratta certo di una novità nella storia della penalità moderna, che non sembra aver mai dismesso la tradizione classista ereditata dal carcere liberale.

In conclusione va detto che la lettura discontinuista della storia recente della penalità, con lo spartiacque della dell'ideale riabilitativo e l'emergere di una approccio attuariale al governo dell'esecuzione penale, sia inadatta a descrivere i mutamenti avvenuti nel sistema dell'esecuzione penale italiana. Dove certamente si ritrovano alcuni fenomeni comuni ad altri sistemi correzionalisti, come quello del net widening. Ma dove la logica welfarista della differenziazione e della allocazione ottimale delle risorse ha finito per consentire la convivenza di orientamenti culturali e pratiche anche contraddittorie: neutralizzazione, rieducazione, reinserimento sociale, giustizia riparativa, mediazione penale e servizio alla comunità, solo per menzionare le principali. Solo apparentemente contraddittorie, ma funzionalmente complementari nel garantire il governo di un sistema dell'esecuzione penale soggetto a ripetute pressioni esterne, di natura politica e mediatica, e continue riforme.

\section{Riferimenti bibliografici}

Albrecht H.J., Schädler W. (1986). Community Service: A new Option in Punishing Offenders in Europe. Freiburg: Max Planck Institute.

Bandini T., Gatti U. (1980). Limiti e contraddizioni dell'opera del criminologo. Rassegna penitenziaria e criminologica, 1,2: 165-174

Baratta A. (1982). Criminologia critica e critica del diritto penale. Bologna: Il mulino.

Barnett R. (1977). Restitution: A new paradigm of criminal justice. Ethics, 87: 279 .

Caputo G., Ciuffoletti S. (2017). Marriage Italian Style. A decryption of Italy and ECtHR's relationship concerning prisoners' rights. In: G. 


\section{Caputo}

Cliquennois, H. de Suremain, a cura di, Monitoring penal policies in Europe.London: Routledge.

Castel R. (1991). From dangerouness to risk. In: Burchell G., Gordon C., Miller P., a cura di, The Foucault effect. Studies in governamentality. Chicago: University of Chicago press

Clemmer D. (1941). The prison community. Boston: The Christopher publishing house.

Cohen S. (1979). The Punitive City: Notes on the Dispersal of Social Control. Contemporary Crises, 3: 339-363

Cohen S. (1985). Visions of Social Control: Crime, Punishment and Classification. Cambridge: Polity press.

Daga L. (2008). Scritti e discorsi 1980-1993. Roma: Ministero della giustizia.

Eglash A. (1977). Beyond restitution: Creative restitution. In: Hudson J., Galaway B., a cura di, Restitution in criminal justice. Lexington: Lexington Books

Feeley M. M., Simon J. (1992). The New Penology: Notes on the Emerging Strategy of Corrections and its Implications. Criminology, 30(4): 449-474.

Foucault M. (1975). Surveiller et punir. Naissance de la prison, Paris: Gallimard (trad. it.: Sorvegliare e punire: la nascita della prigione. Torino: Einaudi, 1993)

Garland D. (1985). Punishment and welfare. Adershot: Gower.

Garland D. (2001). The culture of control. London: Oxford University Press.

Goffman E. (1961). Asylums: Essays on the Social Situation of Mental Patients and Other Inmates. New York: Doubleday.

Ignatieff M. (1978). A just measure of pain. New York: Pantheon Books.

James N. (2018). Risk and Needs Assessment in the Federal Prison System. Congressional Research Service. www.crs.gov

Lemert E. M. (1967). Human deviance, social problems and social control. Englewood Cliffs: Prentice-Hall.

Leonardi F. (2007). Le misure alternative alla detenzione tra reinserimento sociale e abbattimento della recidiva. Rassegna penitenziaria e criminologica, 2: 7. 


\section{Caputo}

Mastrobuoni G., Terlizzese D. (2014). Harsh or Human? Detention Conditions and Recidivism. EIEF Working Papers Series 1413, www.eief.it

Matza D. (1969). Becoming deviant. Englewood Cliffs: Prentice-Hall

Sykes G. M. (1958). The Society of Captives: A Study of a Maximum Security Prison. Princeton: Princeton university press.

Pavarini M. (1997). La criminalità punita. Processi di carcerizzazione nell'Italia del XX secolo. In Violante L., a cura di, La criminalità. Torino: Einaudi.

Pavarini M. (2000). Processi di ri-carcerizzazione e "nuove" teorie giustificative della pena. Rassegna penitenziaria e criminologica, 1, 3: 95-126.

Pratt, J. (2000). Emotive and Ostentatious Punishment. Punishment \& Society, 2, 4: 417-439.

Roberts J. V., Stalans L., Indermaur D., Hough, M. (2003). Penal Populism and Public Opinion. New York: Oxford University Press.

Rothman D. (1971). The discovery of asylum. Boston: Little Brown.

Santoro E. (2004). Carcere e società liberale. Torino: Giappichelli

Santoro E., Tucci R. (2006). L'incidenza dell'affidamento sulla recidiva: prime indicazioni e problemi per una ricerca sistematica. Rassegna penitenziaria e criminologica, 1: 79 ss.

Torrente G. (2014). Il ruolo dell'educatore penitenziario nel processo di criminalizzazione. Studi sulla questione criminale, 1-2: 136.

Von Hirsch A. (1976). Doing justice: the choice of punishments. New York: Hill and Wang. 